\title{
Blood loss reduction in cementless total hip replacement with fibrin spray or bipolar sealer: a randomised controlled trial on ninety five patients
}

\author{
Francesco Falez • Antonio Meo • Gabriele Panegrossi • \\ Fabio Favetti • Filippo La Cava • Filippo Casella
}

Received: 3 March 2013 / Accepted: 17 April 2013 /Published online: 18 May 2013

(C) The Author(s) 2013. This article is published with open access at Springerlink.com

\begin{abstract}
Purpose Several studies have investigated effectiveness of fibrin spray or bipolar sealer to control peri-operative bleeding and reduce the need for blood transfusion, but a direct comparison between the two methods has not been previously performed. We conducted a prospective randomised trial, with standard electrocautery as a control group.

Methods In our investigation, 95 patients were randomised to one of three parallel groups receiving (1) $10 \mathrm{~mL}$ of topical fibrin spray before closure, (2) haemostasis with radiofrequency energy using a bipolar sealer, and (3) standard electrocautery. All patients and staff apart from the surgeons were blinded until data analysis was complete. Peri-operative blood loss has been calculated using a formula described by Ward and Gross (considering estimated patient blood volume, pre- and post-operative haemoglobin and haematocrit levels), with mention of eventual blood re-infusion or transfusion, at given intervals from surgery $(6,24,48,72$ hours).

Results Mean blood loss was lower for both methods investigated, compared to the control group at every time interval considered, although differences were stronger for fibrin spray [Quixil]. Mean blood saving at the given intervals from surgery (6-24-48-72 hours) was respectively $96 \mathrm{ml}$, $129 \mathrm{ml}, 296 \mathrm{ml}$, and $121 \mathrm{ml}$ for bipolar sealer [Aquamantys] and $235 \mathrm{ml}, 368 \mathrm{ml}, 642 \mathrm{ml}$, and $490 \mathrm{ml}$ for fibrin spray. These results are statistically significant $(p=0.05)$ for fibrin
\end{abstract}

Statistical analysis was conducted by Dr. Chiara Pedone (statistician).

F. Falez $(\bowtie) \cdot$ A. Meo • G. Panegrossi · F. Favetti $\cdot$ F. La Cava •

F. Casella

Orthopaedic and Traumatologic Department, S. Spirito Hospital,

L.go Tevere in Sassia nr 1,

00193 Rome, Italy

e-mail: francesco.falez@francescofalez.it spray at every interval compared to control values, while a significance is detectable for bipolar sealer only at 48 hours after surgery.

Conclusions The fibrin spray group had the best performance in terms of blood loss, significantly reduced in comparison with the control group and bipolar sealer group. Blood loss reduction for the bipolar sealer was remarkable only at 48 hours, compared with the control group. Blood loss reduction for fibrin spray was significant at every time interval considered. Differences between the two treatments investigated and the control group narrowed slightly at 72 hours, as an expression of spontaneous homeostasis. Notable is the fact that blood volume saved with fibrin spray at 24 and 48 hours is comparable to the volume of at least one blood unit. A costeffectiveness analysis should be considered in term of expense, biological risks (related to blood transfusion or human-derived products use) and bleeding-related complications.

\section{Introduction}

Primary total hip replacement (THR) is actually one of the most common procedures in orthopaedic elective surgery, but still involves considerable bleeding and a high transfusion rate [1]. Several methods have been used to manage blood loss including intravenous medication, topical application, bipolar sealer or electrocautery.

This investigation compares the use of fibrin spray with bipolar sealer as blood conservation methods in primary cementless THR. Their ability to save blood has been described separately, but no reports have compared them in the THR procedure.

The fibrin spray used (Quixil, Omrix Biopharmaceuticals, Belgium, distributed by Johnson\&Johnson Medical) consists 
of two separate solutions (Active Biological Component and Thrombin) mixed and nebulised at the surgical site; it acts independently of the recipient's coagulation system, unlike tranexamic acid. The bipolar sealer used (Aquamantys Tissue Link Medical, Inc, Dover) is a bipolar, radiofrequency coagulation system delivering a saline solution that produces localised cooling (reducing echar formation).

Estimated blood loss and effecacy of the two methods to reduce peri- and post-operative bleeding compared to standard electrocautery were the endpoints of this investigation.

\section{Patients and methods}

We designed a prospective randomised controlled trial, recruiting our cases from December 2009 through March 2011. It was a single-centre, single-surgeon, single-blind trial, conducted with the approval of the Hospital Ethical Committee.

Each eligible patient (see inclusion and exclusion criteria) was informed about the trial purpose and methods and a specific consent for the use of blood and plasma derived products were collected before definitive recruitment. The study groups (see Table 1) were set up from patients scheduled to undergo elective primary cementless total hip replacement (unilateral).

To determine sample size we considered, in analogy to other studies available in literature [2,3], a difference of at least $30 \%$ in peri-operative blood loss as clinically significant. Considering a mean blood loss post-operatively detected close to $1000 \mathrm{ml}$ in most articles, a blood volume saved between 250 and $300 \mathrm{ml}$ could be considered clinically relevant [4]. Assuming an average blood loss of $1066 \mathrm{ml} \pm 441$ (standard deviation) as in other papers [5], to detect this difference at a $5 \%$ significance level and at $80 \%$ of power, a sample size of 26 patients or more per group was needed. To eliminate possible bias related to the procedure, operations were conducted by the same surgeon, through the same surgical approach (direct lateral in lateral position) and under the same anaesthesia (spinal).

Inclusion criteria were: age between 45 and 75 years; preoperative haemoglobin levels equal or greater than $11 \mathrm{~g} / \mathrm{dL}$; PT, PTT, INR and fibrinogen values within the normal range. The age distribution between groups was similar

Table 1 Study groups

\begin{tabular}{ll}
\hline Study group & Patients \\
\hline Control & 38 \\
Fibrin spray & 31 \\
Bipolar sealer & 26 \\
\hline
\end{tabular}

Table 2 Age distribution between groups

\begin{tabular}{lllll}
\hline Study group & \multicolumn{4}{l}{ Age (years) } \\
\cline { 2 - 5 } & $45-55$ & $56-65$ & $66-75$ & Total \\
\hline Control & 6 & 16 & 16 & 38 \\
Fibrin spray & 5 & 14 & 12 & 31 \\
Bipolar sealer & 4 & 9 & 13 & 26 \\
Overall total & 15 & 39 & 41 & 95 \\
\hline
\end{tabular}

(see Table 2) to reduce a possible bias related to the age of the patients.

Exclusion criteria were: use of anti-platelet aggregating drugs or anticoagulant medications; a known coagulopathy or haemolytic disease; previous thrombo-embolism; previous reaction to blood products; and liver failure. In case of protocol violation, the subject involved was excluded and replaced with a newly-recruited one. The recruitment was stopped when each group had at least 26 patients. Data collection was performed by the attending physician, while statistical analysis was undertaken by an independent statistician.

At admission (usually 24 hours before surgery), patient weight and morphotype (obese, normal, thin, muscular) were recorded to calculate extimated blood volume (EBV) (see Table 3); blood tests for full blood count, electrolytes, liver function, urea and renal function, clotting and crossmatch were obtained.

Each subject received a standard DVT prophylaxis with Enoxaparin 4000 units 12 hours after surgery, paired with elastic stockings and foot-calf pumps immediately after surgery.

Single-blinding of the study was an implicit limit of this investigation, because it is not possible to blind the surgeon to the use of a bipolar sealer or fibrin spray instead of a standard electrocautery. On the other hand, patients and staff members (attending physician, nurses, laboratory technicians) remained blinded.

Patients randomised to the fibrin spray group received topical applications of a combination of two components (supplied in separate vials): (1) a biological active component (BAC) - a concentrated solution of human clottable proteins (fibrinogen and fibronectin) and an antifibrinolytic agent (tranexamic acid); (2) human thrombin-a high

Table 3 Estimated blood volume ( $\mathrm{ml}$ per $\mathrm{kg}$ ) as a function of body habitus, gender and body weight

\begin{tabular}{lll}
\hline Body Habitus & Male & Female \\
\hline Obese & 60 & 55 \\
Thin & 65 & 60 \\
Normal & 70 & 65 \\
Muscular & 75 & 70 \\
\hline
\end{tabular}


potency solution of purified human thrombin and calcium chloride for activation of clotting of the final combined product.

We prefer application of the fibrin spray at the end of the procedure, before closure; during surgery, largest vessels need, on our advice, standard electrocautery or ligature. Moreover, the thin layer derived from application may be altered from lavages and swabs before the procedure was accomplished.

Patients randomised to the bipolar sealer group received a continuous haemostatic action, using the device in a painting motion or as spot treatment for bleeding vessels.

The bipolar sealer provides haemostasis at $100{ }^{\circ} \mathrm{C}$ or less because it delivers radiofrequency to saline; this temperature is lower than those of conventional electrocautery and does not char or burn tissue during haemostatic sealing and coagulation of soft tissue.

An articular drainage activated by means of low pressure and three non-return valves (Bellovac ${ }^{\mathrm{TM}}$ Astra Tech) was used at the end of each procedure; re-infusion was planned six hours after completion of surgery (recording blood volume collected), and each patient underwent full blood count prior to re-infusion.

Full blood count was repeated at given intervals (24, 48 and 72 hours) and eventual further blood transfusion was recorded.

To obtain the peri-operative blood loss we applied a formula described by Jeffrey B. Gross (corrected for dilution in its logarithmic version) [6] considering the blood volume eventually re-infused: $\mathbf{V}_{\mathbf{L}}=\mathbf{E B V} \times \mathbf{I}_{\mathbf{n}}\left(\mathbf{H}_{\mathbf{0}} / \mathbf{H}_{\mathbf{F}}\right)+\mathbf{H}_{\mathbf{T}}$ which calculates overall blood loss $\left(\mathrm{V}_{\mathrm{L}}\right)$ in relation to estimated blood volume (EBV, determined from gender, weight and body habitus as reported in Table 3), decreasing in haemoglobin concentration (or haematocrit) $\left[\mathrm{l}_{\mathrm{n}}\left(\mathrm{H}_{0} / \mathrm{H}_{\mathrm{F}}\right)\right]$, where $l_{n}$ is the natural logarithm, $H_{0}$ is pre-operative haemoglobin concentration and $\mathrm{H}_{\mathrm{F}}$ is haemoglobin concentration at a given interval and volume of blood transfused $\left(\mathrm{H}_{\mathrm{T}}\right)$ from drainage (within the first six hours postoperatively) or blood units (in $\mathrm{ml}$ ).

Data for the three treatments (fibrin spray, bipolar sealer, standard electrocautery) were analysed performing a pairwise comparison between the sample means of each active treatment and control, using the Student's t-test. Data collection and analysis was performed with the Microsoft Excel tool (Microsoft Corporation, Redmond, Washington) and software R2.13.1.

\section{Results}

No adverse effects were detected during the trial, enabling us to complete patient recruiting and data collection. Patients excluded from the study because of protocol violation (one received Dabigatran as oral prophylaxis against DVT, three missed one or more total blood count, two received a non-recorded volume of re-infused blood from drainage) were replaced by newly recruited subjects. When at least 26 cases had been collected in all groups, the recruitment was stopped and data analysis was performed. A total of 95 patients were available for data analysis. Calculated blood loss at the given intervals is summarised in Table 4 (mean values).

Control group (standard electrocautery) showed a mean post-operative blood loss of $960 \mathrm{ml}$ at 6 hours, $1573 \mathrm{ml}$ at 24 hours, $2016 \mathrm{ml}$ at 48 hours and $1862 \mathrm{ml}$ at 72 hours. Comparing the fibrin spray group with the control group, we detected a blood savings at 6, 24, 48 and 72 hours of 235, 368, 642 and $490 \mathrm{ml}$, respectively. Comparing the bipolar sealer group with the control group, we detected a blood savings at 6 , 24, 48 and 72 hours, of 96, 129, 296 and $121 \mathrm{ml}$, respectively.

Considering each given interval from surgery, we calculated the statistical significance (Student's t-test, interval of confidence $95 \%$ ). These differences were statistically significant (Student's t-test, $p=0.05$ ) in all intervals considered between the fibrin spray group and control, while for comparison between the bipolar sealer and control groups a significant difference was observed only at 48 hours (see Table 5).

\section{Discussion}

As often underlined in clinical practice, blood is a finite resource that needs careful and uninterrupted review of possible areas as to where to improve its use (minimising waste and reducing clinical demand). Total hip replacement, due to its worldwide application (with a progressive increase in number of cases per year) and its peri-operative blood transfusion rate (still considerable, even in the presence of minimally invasive surgery), is probably one of the most remarkable fields of application where blood saving procedures may realise significant results.

We strongly believe that in surgical practice blood loss calculated from suction drains and swabs underestimate the real peri-operative bleeding (not considering hidden losses, i.e. post-operative haematoma); this underestimation

Table 4 Calculated blood loss for each group at given intervals

\begin{tabular}{llccc}
\hline Study group & \multicolumn{4}{c}{ Blood Loss (ml) at given intervals } \\
\cline { 2 - 5 } & $6 \mathrm{~h}$ & $24 \mathrm{~h}$ & $48 \mathrm{~h}$ & $72 \mathrm{~h}$ \\
\hline Control (38 cases) & 960 & 1573 & 2016 & 1862 \\
Fibrin spray (31 cases) & 725 & 1205 & 1373 & \\
Bipolar sealer (26 cases) & 864 & 1444 & 1719 & 1741 \\
Mean & 857 & 1417 & 1694 & \\
\hline
\end{tabular}


Table 5 Output of statistical comparison between results achieved in treatment groups ( $F S$ fibrin spray, $B S$ bipolar sealer) and control group (Student's t-test, $95 \%$ confidence interval). The null hypothesis states that the two treatments have the same results as control

\begin{tabular}{lllll}
\hline $\begin{array}{l}\text { Groups } \\
\text { compared }\end{array}$ & Time after surgery & & & \\
\cline { 2 - 5 } & $6 \mathrm{~h}$ & $24 \mathrm{~h}$ & $48 \mathrm{~h}$ & $72 \mathrm{~h}$ \\
\hline FS-Control & $t=2.1688$ & $t=2.906$ & $t=4.1706$ & $t=3.1242$ \\
& $d f=58$ & $d f=67$ & $d f=58$ & $d f=61$ \\
& $p$-value $<0.05(0.03421)$ & $p$-value $<0.05(0.004957)$ & $p$-value $<0.05$ & $(0.0001028)$ \\
& Null-hypothesis rejected & Null-hypothesis rejected & Null-hypothesis rejected & Null-hypothesis rejected \\
& $t=0.8406$ & $t=1.0762$ & $t=2.0899$ & $t=0.7725$ \\
BS-Control & $d f=62$ & $d f=53$ & $d f=61$ \\
& $d f=56$ & $p$-value $>0.05(0,286)$ & $p$-value $<0.05(0.04144)$ & $p$-value $>0.05(0.4428)$ \\
& $p$-value $>0.05(0.4042)$ & Null-hypothesis can't be & Null-hypothesis rejected & Null-hypothesis can't be \\
& Null-hypothesis can't be & rejected & & rejed
\end{tabular}

extends up to $26 \%$ in total hip replacement, as observed previously [7]. This is the reason why we use a validated formula to estimate blood loss; that formula, in the natural logarithmic fashion used in our study, is probably not suited for routine use in O.R. to estimate volume to be replaced, but it has been shown to correspond accurately to measured blood losses in humans $[8,9]$ and animals.

Most previous studies have detected blood loss in a single post-operative period (often immediately after surgery) and their results reflect this aspect with a limited total volume of blood lost; in our study the total blood loss appears to be higher because its detection has been extended through the first 72 hours after surgery (on the other hand, our mean blood loss values at six hours after surgery are similar to results reported in the literature). Extending our investigation through 72 hours after surgery (in consideration of possible postoperative bleeding), we must consider the possibility of re-infusion from drainage or transfusion during that period (even if there is a potential bias derived from a spontaneous subjective response to anaemia).

Two more considerations should be taken into account resulting from of post-operative re-infusion with autologus blood. Specifically, there may be a deficit in haemoglobin concentration due to haemolysis in the re-infused blood (however, in the presence of low suction pressure devices, appreciable haemolysis has not been detected in a previous study focussed on hidden blood loss in total hip and knee surgery [7]). A randomised controlled blinded prospective study, analysing the potential benefit using an autologus blood transfusion system, detected no significant advantages (in post-operative haemoglobin levels, number of homologous blood transfusions, clinical outcomes) compared with patients that received no drain [10].

As reported in our results, the ability of fibrin spray in bleeding control seems to outperform bipolar sealer at every time interval considered. We interpret this behaviour as a limit of the bipolar sealer in reducing local bleeding when blood pressure and body temperature return to normal values in the post-operative period (even in the presence of an extensive use of this device through the different layers of the surgical approach).

Some investigations, to confirm this interpretation, have changed bipolar sealer perspectives detecting no significant differences between the treatment and control groups in terms of the need for blood transfusions or overall blood loss [11, 12].

On the other hand, the fibrin film sprayed on the surface may act even a few hours after the end of surgery and may prevent bleeding from the bone surface (where the use of the Bipolar sealer is not recommended).

The mean blood savings compared to control treatment was, in the presence of the fibrin spray, between 235 and $642 \mathrm{ml}$. It is remarkable in terms of clinical significance (a reduction of one red-cell unit for transfusion) and statistical significance $(p<0.05)$, more than observed with the Bipolar sealer (included between 96 and $296 \mathrm{ml}$ ), even if a direct comparison between the two methods was not an objective of the present study.

The potential benefit derived from avoiding one or more transfusion may exceed the biological risk generically ascribed to pathogens. Most previous studies have reported an increased risk of postoperative infections including pneumonia, short-term mortality, length of hospital stay and systemic inflammatory response syndrome in patients treated with one or more blood transfusion [13]. A costeffectiveness analysis, finally, should lead surgeons to take into account the impact of additional devices in routine practice, e.g. even in this case, the potential benefit derived from the fibrin spray seems to justify the additional charges.

Previous studies, however, have underlined how cheaper strategies (such as tranexamic acid, TA), may surpass the fibrin spray in term of costs. The therapeutic dose of TA set at $10 \mathrm{mg} / \mathrm{kg}$ at induction of anaesthesia will cost in our country 
about $8 €$; a Quixil fibrin spray set (two $5 \mathrm{ml}$ vials and nebuliser) costs between $450 €$ and 675,00€ [4]; an Aquamantys Bipolar Sealer set costs up to $1440,00 €$.

But considering tranexamic acid, the manufacturer does not give a recommended dose for surgery and most actual protocols are only based upon clinical observation, giving potential risks of under- or over-dosage. In the literature we can find studies with single doses of $10 \mathrm{mg} / \mathrm{kg}$ at the induction of anaesthesia [4], higher doses [14] or multiple doses [15]. Moreover, the same dose can have different effecacy if administrated at the induction or at the end of surgery [16], and it is difficult to state whether its effect continues even at a period of six or more hours after surgery.

Fibrin spray and of course bipolar sealer, if correctly used, are definitely topical treatments, with minimal or no risks of interference with coagulation away from the surgical site (unlike tranexamic acid, that is based on an intra-venous administration) and both of them act independently from the recipient's coagulation system (while tranexamic acid acts mainly by reducing fibrinolysis, inhibiting plasminogen with a competitive action, depending on the patient's coagulation for clot formation). These mechanisms of action make fibrin spray more selective for the surgical site and potentially expand their application to the patient affected by coagulopathy.

A potential detriment to the use of fibrin spray could be the potential risk of disease transmission; processing and screening are able to eliminate all pathogens, but concern may remain relating to unknown vectors.

A new frontier could be represented by autologous fibrin sealants prepared from blood donated prior to surgery through centrifugation, after a freeze-and-thaw process repeated twice and again centrifuged, and thus obtaining an autologous cryoprecipitate (to be mixed in a double-syringe spray with a second solution composed of thrombin, calcium chloride and aprotinin). This autologous fibrin spray, used in hip surgery, has shown statistically significant results in bleeding control [17].

Open Access This article is distributed under the terms of the Creative Commons Attribution License which permits any use, distribution, and reproduction in any medium, provided the original author(s) and the source are credited.

\section{References}

1. Stokes ME, Ye X, Shah M, Mercaldi K, Reynolds MW, Rupnow MFT, Hammond J (2011) Impact of bleeding-related complications and/or blood product transfusions on hospital costs in inpatient surgical patients. BMC Health Service Research 11:135-147
2. Wang GJ (2003) Fibrin sealant reduces perioperative blood loss in total hip replacement. J LongTerm Eff Med Implants 13(5):399411

3. Marulanda GA, Ragland P, Mont MA, Flowers NB (2006) Use of a bipolar sealer device for hemostasis In total hip arthroplasty. Paper no. 382, presented at the American Academy of Orthopaedic Surgeons, Annual Meeting, Chicago

4. McConnell JS, Shewale S, Munro NA, Shah K, Dakin AH, Kinninmonth AWG (2011) Reduction of blood loss in primary hip arthroplasty with tranexamic acid or fibrin spray. A randomized controlled trial. Acta Orthop 82(6):660-663

5. Johansson T, Lisander B, Ivarsson I (1999) Mild hypothermia does not increase blood loss during total hip arthroplasty. Acta Anaesthesiol Scand 43(10):1005-1010

6. Gross JB (1983) Estimating allowable blood loss: corrected for dilution. Anesthesiology 58:277-280

7. Sehat K, Evans R, Newman (2004) Hidden blood loss following hip and knee arthroplasty. Correct management of blood loss should take hidden loss into account. J Bone Joint Surg (Br) 86:561-565

8. Bourke DL, Smith TC (1974) Estimating allowable hemodilution. Anesthesiology 41:609-612

9. Ward CF, Meathe EA, Benumof JF, Trousdale F (1980) A computer nomogram for blood loss replacement. Anesthesiology 53:S126

10. Horstmann WG, Kuipers BM, Slappendel R, Castelein RM, Kollen BJ, Vertheyen CCPM (2012) Postoperative autologous blood transfusion drain or no drain in primary total hip arthroplasty? A randomised controlled trial. Int Orthop 36(10):2033-2039

11. Barsoum WK, Klika AK, Murray TG, Higuera C, Lee HH, Krebs VE (2011) Prospective randomized evaluation of the need for blood transfusion during primary total hip arthroplasty with use of a bipolar sealer. J Bone Joint Surg Am 16;93(6):5138

12. Zeh A, Messer J, Davis J, Vasarhelyi A, Wohlrab D (2010) The Aquamantys system - an alternative to reduce blood loss in primary total hip arthroplasty? J Arthroplasty 25(7):10721077

13. Pedersen AB, Mehnert F, Overgaard S, Johnsen SP (2009) Allogenic blood transfusion and prognosis following THR: a population-based follow up study. BMC Musculoskeletal Disord 10:167-178

14. Johansson T, Pettersson LG, Lisandr B (2005) Tranexamic acid in total hip arthroplasty saves blood and money: a randomized, double-blind study in 100 patients. Acta Orthop 76(3):314319

15. Niskanen RO, Korkala OL (2005) Tranexamic acid reduces blood loss in cemented hip arthroplasty: a randomized, double-blinded study of 39 patients with osteoarthritis. Acta Orthop 76(6):829 832

16. Benoni G, Fredin H, Knebel R, Nilsson P (2011) Blood conservation with tranexamic acid in total hip arthroplasty a randomized, double-blind study in 40 primary operations. Acta Orthop Scand 72(5):442-444

17. Mawatari M, Higo T, Tsutsumi Y, Shigematsu M, Hotokebuchi T (2006) Effectiveness of autologous fibrin tissue adhesive in reducing postoperative blood loss during total hip arthroplasty: a prospective randomized study of 100 cases. J Orthop Surg 14(2):117-121 